\title{
A tunable source of coherent terahertz radiation driven by the microbunched electron beam
}

\author{
Huibo Zhang ${ }^{1}$, Ivan Konoplev ${ }^{1}$, George Doucas ${ }^{1}$ \\ ${ }^{1}$ John Adams Institute for Accelerator Science, Department of Physics, University of Oxford, \\ Oxford, OX1 3RH, UK \\ E-mail: ivan.konoplev@physics.ox.ac.uk
}

\begin{abstract}
The studies of coherent terahertz (THz) tunable Smith-Purcell radiation source driven by the microbunched electron beam are presented. We discuss the conceptual design of a high power and broadly tunable $\mathrm{THz}$ radiation source capable of bridging the THztechnological gap. The results of the numerical and analytical studies using different software packages, models and approaches are shown and good agreement between the results is demonstrated. The spectra of the coherent Smith-Purcell radiation (cSPr) excited by a single bunch and train of the microbunches are compared and the excitation of the harmonics of cSPr is discussed. It is shown that varying the distance between microbunches the power and the frequency of the output cSPr can be controlled.
\end{abstract}

\section{Introduction}

The electromagnetic radiation in the $\mathrm{THz}$ frequency range bridges the gap between microwaves (frequency down to $0.1 \mathrm{THz}$ ) and far infrared (up to $10 \mathrm{THz}$ ) in the electromagnetic (EM) spectrum. Due to its features, such as: (a) its significant penetration depth in many non-metallic and non-polar materials; (b) strong absorption by some organic materials and by water at certain frequencies $[1,2,3]$, the THz region of the EM radiation has acquired great importance over a wide range of topics in research and industry. The $\mathrm{THz}$ radiation based solution and technology have been used for security and defense applications, such as the detection of explosives [4, 5], personnel and security mail screening [6], and the detection of noxious gases [7, 8]. It has also been used in cultural heritage investigations [9], communications [10], and many other areas [11].

With the recent developments in accelerator science and technology, there has been a growing interest in the sources of $\mathrm{THz}$ radiation driven by electron linear accelerators (linacs) $[12,13,14,15]$. There are a number of advantages of using linacs. For example, the spacecharge effects can be mitigated in case of a relativistic electron beam allowing high power radiation to be generated. Also the application of energy recovery [16, 17, 18] has been explored to increase the overall efficiency of the system. The ultimate features of such sources which make them attractive include tunability over a broad frequency $(0.1 \mathrm{THz}$ to $10 \mathrm{THz})$ and power ranges. 
The bunch length of a femtosecond ( $\mathrm{fs}$ ) long relativistic electron beam is such that the radiation generated by the beam is coherent in the $\mathrm{THz}$ frequency range, i.e. the bunch length of the beam is shorter than the radiation wavelength, and the energy of the coherent radiation is proportional to the square of number of the electrons in the bunch [19, 20]. Due to the space-charge effects, it is difficult to drive a fs-long beam with a high charge (above $1 \mathrm{nC}$ ) when the beam has a relatively low energy $(<10 \mathrm{MeV})$. To overcome this limitation, a train of the microbunches (rather than a single bunch) can be used [21, 22, 23]. Recently, several methods of generating the trains of relativistic electron microbunches with a picosecond (ps) or sub-picosecond periodicity have been investigated. These include the laser based methods [24, 25, 26, 27, 21] (direct beam current/beam energy modulations at the photocathode), dielectric wakefield induced modulation [28], the transverse to longitudinal phase space exchange method [29], and the beam modulation in a plasma via the selfmodulation instability (SMI) [30, 31, 22].

The microbunched beam can be used to generate the $\mathrm{THz}$ radiation with the basic principle is that the kinetic energy of electrons is converted into the energy of electromagnetic waves [15]. In particular one of the methods which allows to generate coherent $\mathrm{THz}$ radiation and use the electron beam energy recovery (at the final stage) is based on the interaction of the electron beam and a periodic structure leading to the excitation of the coherent Smith Purcell radiation (cSPr). Usually the Smith-Purcell radiation is generated when an electron beam propagates in the vicinity of a periodic metallic grating and it was first studied by S. J. Smith and E. M. Purcell in 1953 [32]. A Huygens construction law can be used to illustrate the dispersion relation in the far-field region, which is as follows:

$$
\lambda=\frac{l}{n}\left(\frac{1}{\beta}-\cos \theta\right)
$$

where $\lambda$ is the radiation wavelength, $l$ is the period of the grating, $n$ is the radiation order, $\beta=v / c$ is the relativistic factor of the beam, and $\theta$ is the observation angle relative to the beam propagation direction. Selecting the grating period in the millimetre $(\mathrm{mm})$ and sub-mm range, the $\mathrm{THz}$ radiation can be observed from a fs-long relativistic electron beam. When the electron beam is modulated, the tunability of the $\mathrm{THz}$ radiation source can be achieved through varying the microbunch periodicity. There is no need for an external cavity (as used in many conventional vacuum oscillators) allowing continuous tuning of the source over a wide range of frequencies. Besides, Smith-Purcell radiation mechanism causes no beam loss making it possible as mentioned above the application of the energy recovery to increase the overall efficiency of the source.

In the following sections, the generation of $\mathrm{THz}$ radiation using the microbunched electron beam is investigated. The effect of the microbunching of the beam on the bunch form factor is discussed in section 2. Particle-in-Cell (PIC) software packages VSim and CST are used to simulate coherent Smith-Purcell radiation generated by a single fs-long electron bunch and by the microbunched electron beam. The results of the numerical and semi-analytical studies are presented in sections 3 and 4 . Section 5 summarizes this work and the concept of the $\mathrm{THz}$ source of coherent radiation driven by energy recovery linac is briefly described. 


\section{Analysis of the bunch form factor}

The energy of the Smith-Purcell radiation per unit solid angle emitted by a bunch with $N$ electrons is given in the references $[33,34,35]$, as:

$$
\left(\frac{d I}{d \Omega}\right)_{N}=\left(\frac{d I}{d \Omega}\right)_{1}\left[N S_{\text {inc }}+N(N-1) S_{c o h}\right]
$$

where $(d I / d \Omega)_{N}$ is the radiation energy per solid angle $\Omega$ from $N$ electrons, $(d I / d \Omega)_{1}$ is the radiation energy from a single electron, $S_{i n c}$ and $S_{c o h}$ are the incoherent and coherent radiation form factors, which are defined by the charge distribution of the electrons in the bunch. Normally the transverse dimension of the bunch can be focused to be much smaller than the operating wavelength and the influence of the transverse profile can be neglected. In this case:

$$
\left(\frac{d I}{d \Omega}\right)_{N} \approx\left(\frac{d I}{d \Omega}\right)_{1}\left[N+N(N-1)|F(v)|^{2}\right]
$$

where $|F(v)|=\left|\int_{-\infty}^{+\infty} T(t) \exp (-j 2 \pi v t) d t\right|$ is the longitudinal bunch form factor. The first term in the square brackets of the equation 3 describes the incoherent radiation and it is proportional to $N$, while the second term represents the coherent radiation, proportional to $N^{2}$. A typical electron bunch (0.1-1 nC) has $N \approx 10^{9}-10^{10}$ electrons making the energy of the coherent radiation is much higher than that of the incoherent radiation if $|F(v)|$ is of order unity.

Considering a train of the microbunches, i.e. an electron beam consisting of $M$ identical microbunches equally separated by an interval $\Delta t$ with a repetitive longitudinal temporal profile $T(t-n \Delta t), n=0,1 \ldots, M-1$, the form factor $|F(v)|$ is given by:

$$
\begin{aligned}
& |F(v)|^{2}=\left|F_{1}(v)\right|^{2} G_{M}(v, \Delta t) \\
& G_{M}(v, \Delta t)=\frac{1}{M^{2}} \frac{\sin ^{2}(M \pi v \Delta t)}{\sin ^{2}(\pi v \Delta t)}
\end{aligned}
$$

where $\left|F_{1}(v)\right|$ is the form factor of a single microbunch and $G_{M}(v, \Delta t)$ is a modulation function depending on the observation frequency $v$ and the microbunch spacing $\Delta t$. For a single Gaussian microbunch $(M=1)$ with RMS longitudinal length $\sigma$ (in units of time), the square of the form factor $|F(v)|^{2}$ becomes $\exp \left[-(2 \pi v \sigma)^{2}\right]$ which indicates that the spectral width of $|F(v)|^{2}$ is getting wider by reducing the bunch length $\sigma$.

The comparison of the square of the form factors for a single bunch ( $0.1 \mathrm{ps}$ length) and a train of the microbunches is shown in figure 1. The total charge of the trains is the same and it is distributed evenly between equally spaced ( $1 \mathrm{ps}$ ) identical microbunches of the length 0.1 ps . Unlike the broad spectrum of a single bunch, the form factor of the microbunch train has peaks at the fundamental beam current frequency $v_{b}(1 \mathrm{THz})$ and its high harmonics located at $n v_{b}(n \geq 2)$. The spectral width $\Delta v$ of the form factor at the frequency $v_{b}$ is proportional to $v_{b} / M$, i.e. an increase of the number of the microbunches in the train leads to the narrowing of the peaks of the form factor. One also notes that for the cSPr spectrum in the far-field region, the spectral width at a single frequency $v$ is around $v / N_{g r}$ where $N_{g r}$ is the number of the grating periods (on the order of several hundreds). The number of the microbunches $M$ 


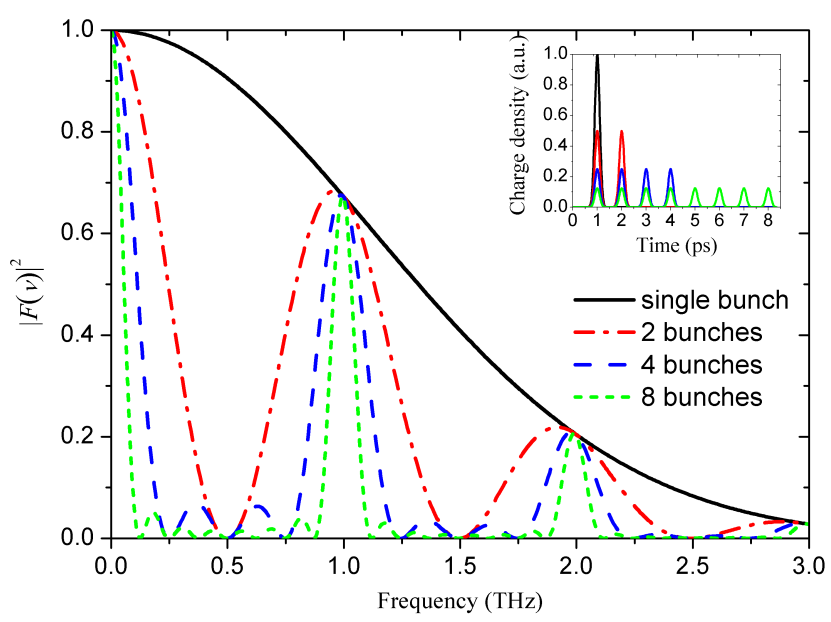

Figure 1. Comparison of the frequency dependence of $|F(v)|^{2}$ observed for a single bunch (solid line) and a train of the microbunches consisting of 2 (dash-dot line), 4 (dashed line) and 8 (dotted line) microbunches with the microbunch separation of $1 \mathrm{ps}$ (inset is the corresponding charge density distribution).

in the electron beam generated as discussed above is normally well below a hundred. In this case the effect of the microbunching on the spectral width of the cSPr in the far-field region can be neglected.

\section{Numerical studies of cSPr in the near-field region}

VSim is a flexible, cross-platform computational software tool for running electromagnetic and plasma simulations [36]. Simulations in three dimensions (3D) are normally computationally intensive. All the VSim simulations discussed in this paper were performed in two dimensions (2D) by assuming an infinite wide grating along the $y$ direction (figure 2, $y$ direction is perpendicular to the $x z$-plane). We deal with the system which has open boundaries and a module called perfectly matched layer (PML) in VSim was used. The PML is an artificial absorbing layer which is designed to absorb the incident waves. For particles, an absorbing box was used to remove the particles incident upon the surface from the simulation.

Figure 2 is a schematic of the VSim model which shows all the significant dimensions. The simulation box is $23 \mathrm{~mm}$ (longitudinal direction) by $12 \mathrm{~mm}$ (transverse direction) and it is divided into 2758 by 2058 cells, respectively. Since the transverse dimension of the beam and the beam-grating separation are much smaller than the transverse dimensions of the gratings, the $2 \mathrm{D}$ approximation for the numerical model is considered to be reasonable. The beam and grating parameters are shown in table 1.

To generate Smith-Purcell radiation, the beam passed above the grating along the $z$ direction and the distance between beam center and the grating was $0.5 \mathrm{~mm}$. The beam profiles were set to be Gaussian in both the longitudinal $(z)$ and transverse $(x)$ directions. The field monitor, which was used to record the magnetic field component $B_{y}$ of the radiation, was 


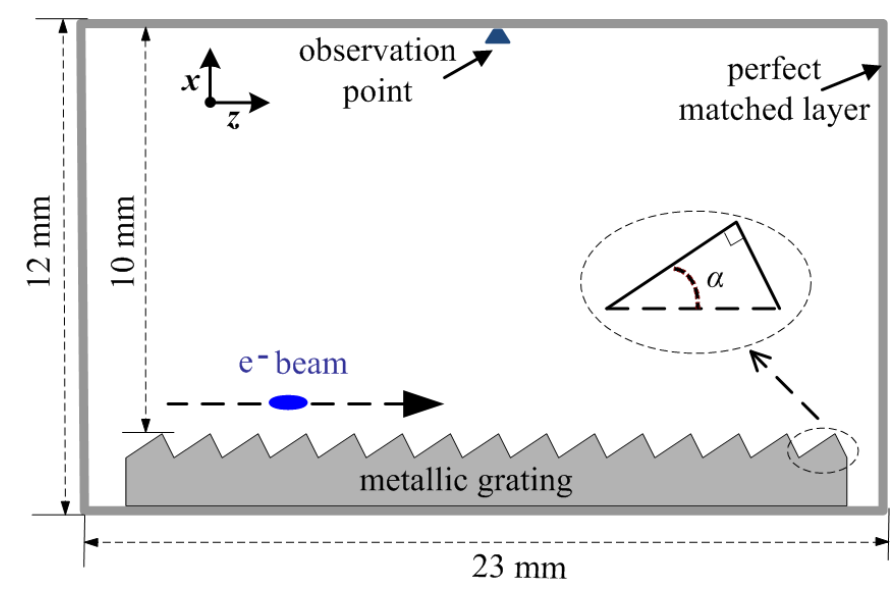

Figure 2. Schematic diagram of the VSim simulation box showing the coordinate system, grating, electron beam and its direction of the propagation, a single observation point and the essential dimensions.

Table 1. Beam and grating parameters.

\begin{tabular}{lcl}
\hline Description & Parameters & Value \\
\hline Beam energy & $E$ & $50 \mathrm{MeV}$ \\
Beam charge & $Q$ & $1 \mathrm{nC}$ \\
Beam longitudinal size & $\sigma_{l}$ & $50 \mu \mathrm{m}(0.167 \mathrm{ps})$ \\
Beam transverse size & $\sigma_{t}$ & $30 \mu \mathrm{m}(0.1 \mathrm{ps})$ \\
Grating period & $l$ & $500 \mu \mathrm{m}$ \\
Facet number & $N_{f}$ & 25 \\
Facet angle & $\alpha$ & $30^{\circ}$ \\
Beam grating distance & $d$ & $500 \mu \mathrm{m}$ \\
\hline
\end{tabular}

positioned $10 \mathrm{~mm}$ above the grating center (near-field region) and at $90^{\circ}$ to its surface.

Each time the beam passes above grating's facet, the discontinuity of the facet perturbes the propagation of the induced surface current resulting in generating the electromagnetic radiation which propagates into the space away from the grating [35]. In the far-field region, the radiation waves from the periodic array of the grating's facets will interfere forming the dispersion patterns defined by the relation (equation 1) in $x z$-plane.

The evolution of the Smith-Purcell radiation in the near field region while the beam propagates above the grating is shown in figure 3 . The contour plot shows the snapshot of the magnetic field $B_{y}$ at the time $t$ of $40 \mathrm{ps}$. The figure was observed by subtracting the signal from a blank target (an identical metal piece with no corrugations on the surface) from the total signal observed from the grating. The subtraction allows removing the beam's self-fields and the fields associated with the diffraction radiation from the edges of the target thus yielding only the cSPr generated and the surface fields on the grating excited by the beam.

One notes that in the near-field zone the interference of the radiation can be neglected 


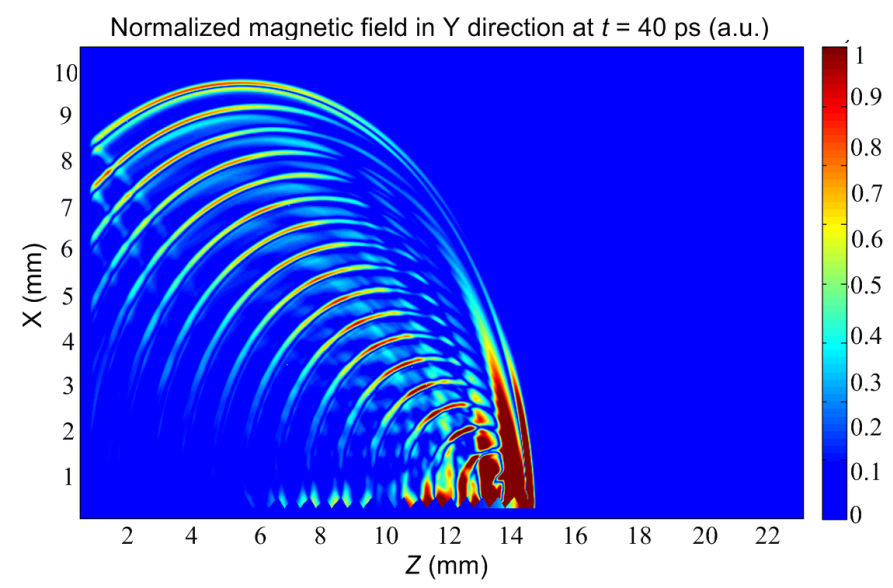

Figure 3. Snapshot of the distribution of the magnetic field $B_{y}$ component of the electromagnetic radiation generated when the electron beam propagates along the grating. The snapshot is made at the simulation time $t=40 \mathrm{ps}$.

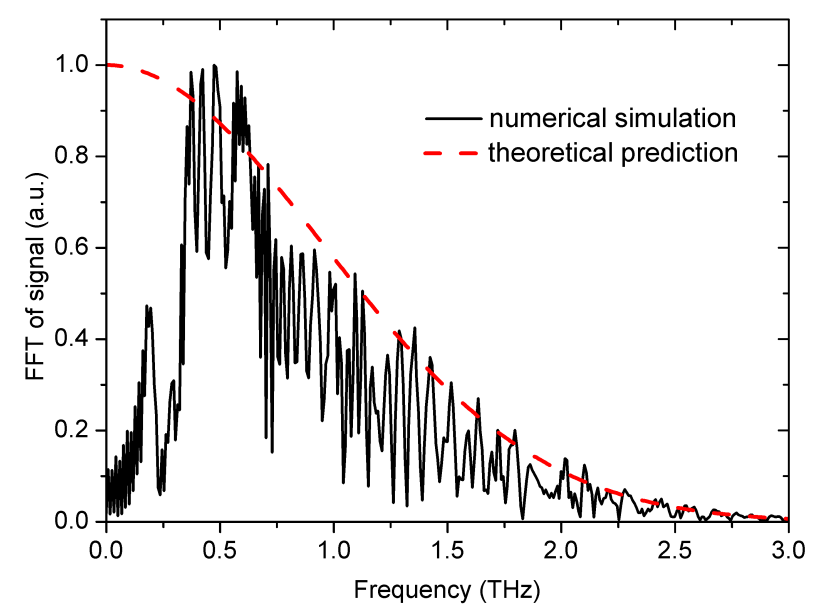

Figure 4. Comparison of the form factors obtained from the theoretical predictions (dashed line) and from the numerical simulations (solid line).

and without loss of generality while still avoiding "edge effects", the radiation observed above the center of the grating (figure 2) has been chosen for the analysis. Figure 4 shows the comparison of the form factors obtained from theoretical predictions (dashed line) and numerical simulations (solid line). The dashed line was calculated using $|F(v)|=$ $\exp \left[-2\left(\pi v \sigma_{l}\right)^{2}\right]$ for a Gaussian bunch. The solid line was the Fourier transform of the cSPr signal $B_{y}$ observed in the near-field region using VSim. There are two main features in figure 4, namely, the exponential decay of the amplitude of the spectrum from $0.5 \mathrm{THz}$ to $3 \mathrm{THz}$ (prediction shows similar pattern) and the drop of radiation energy at the "cut-off" frequency for Smith-Purcell radiation $\left(0.3 \mathrm{THz}\right.$, angle $\left.\theta=180^{\circ}\right)$. The last feature is due to the subtraction of the total signal from the signal observed from blank target.

Using the same beam parameters and splitting the total charge into $M$ identical 


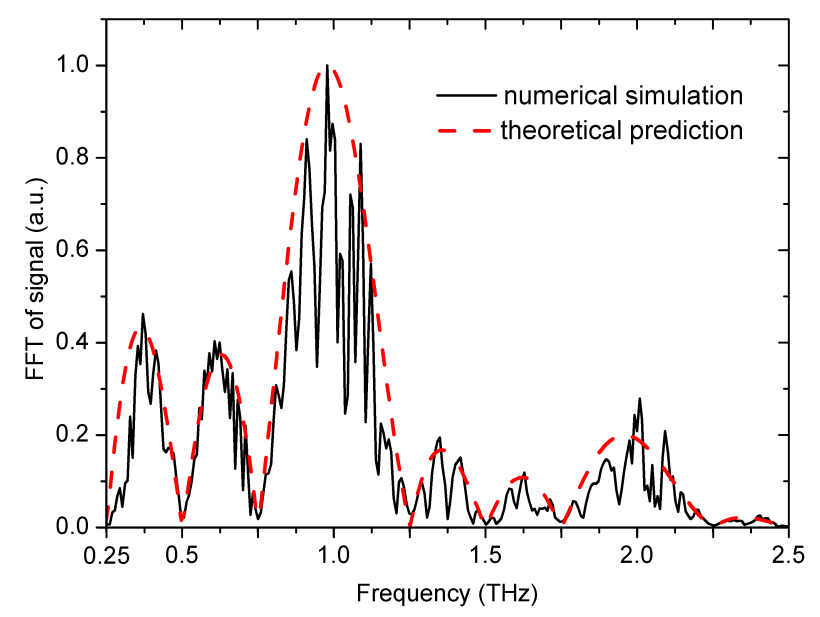

Figure 5. Comparison of the form factors obtained from the theoretical predictions (dashed lines) and the Fourier transform of the cSPr signal $B_{y}$ observed from VSim simulations (solid lines) for the beam consisting of 4 identical microbunches with the microbunch separation of 1 ps.

microbunches ( $M=4$ in this case), simulations were repeated. The Fourier transform of the radiated signal observed at the angular position of $90^{\circ}$ for the microbunch trains with the separations of $1 \mathrm{ps}$ is shown in figure 5. The spectral peaks of the radiation are located at 1 $\mathrm{THz}$ as expected. As a remark to figures 4 and 5, we note that traditionally cSPr is investigated in the far-field region $[33,34,35]$, i.e. the dispersion relation (equation 1) is fulfilled while the spectra lines (black line) in figures 4 and 5 are evaluated from the signals measured in the near-field and the spectrum of the measured signal contains the whole spectrum of the cSPr generated by the surface currents.

\section{Numerical studies of cSPr in the far-field region}

The PIC simulations have also been carried out using the CST Particle Studio (PS). This is another fully relativistic, self-consistent PIC solver which takes into account the spacecharge effects and EM fields radiated by the beam. The studies were carried out to investigate the cSPr in the far-field region and compare it with the numerical results from the VSim and analytical predictions. To validate the studies, it is a good practice to compare results obtained using different numerical tools. The available CST Particle Studio and workstation resources have allowed us to carry out the studies in three dimensions fully consistently using the time domain PIC solver. To perform the numerical studies, the open boundary conditions were applied in the CST simulations. The CST PIC solver allows the far-field radiation to be recorded by the far-field probes from the field distribution in the near-field zone (build-in EM integration capability), which enables investigation of the radiation properties in the far-field region and direct comparison with the analytical results.

The schematics of the numerical model is shown in figure 6 , where $\vec{k}$ is the wave vector of 
the radiation, $\phi$ and $\theta$ is the azimuthal and polar angles (spherical coordinate system) relative to the beam axis. The probes used for the recording of the electromagnetic fields in the farfield region, were positioned at $1 \mathrm{~m}$ away from the center of the grating (origin ' $o$ ') at the $\theta$ angles from $60^{\circ}$ to $150^{\circ}$ with the interval of $1^{\circ}$ (91 points). All the probes were in the plane $\phi=0$.

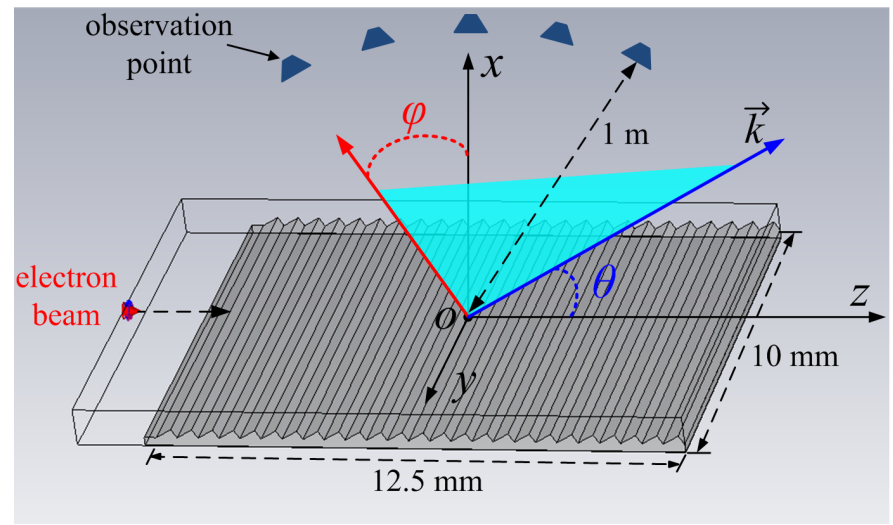

Figure 6. The schematic (not-to-scale) of the CST simulation showing periodic grating, angular position of the detectors, and the beam trajectory.

Adaptive meshing was used in the model, i.e. the mesh size was small in the regions where the fine structures (grating) appear. The smallest mesh size was $1.7 \mu \mathrm{m}$ (in the $x$ direction) and the largest mesh size was $15 \mu \mathrm{m}$ (in the $y$ direction). Both dimensions are much smaller than the radiation wavelengths of the interest (from $150 \mu \mathrm{m}$ to $600 \mu \mathrm{m}$ ).

As in previous section the numerical studies have been started with the simulation of the cSPr generation by a single electron bunch (the parameters as shown in Table 1). The signal waveform observed at $\theta=80^{\circ}$ and its corresponding Fourier transform are shown in figures 7(a) and 7(b), respectively. The waveform shown in figure 7(a) can be split into 4 partial signals: the first is caused by the self-fields of the beam, the second is the diffraction radiation signal from the edge of the grating, the main part of the signal (indicated by 3 ) is the cSPr, and the tail of the waveform (indicated by 4) is due to the reflection from the boundaries (not a perfect open structure). As before to remove the influence of the beam self-fields and the diffraction radiation at the edge of the grating, the Smith-Purcell radiation signal was obtained by subtracting the signal from the blank target from the total signal observed from the grating. In figure 7(b), the frequencies of the first three harmonics of the Smith-Purcell radiation are shown and located at $0.73 \mathrm{THz}, 1.457 \mathrm{THz}$ and $2.2 \mathrm{THz}$ which agree well with the frequencies $(0.726 \mathrm{THz}, 1.452 \mathrm{THz}, 2.178 \mathrm{THz})$ obtained from the dispersion relation. As expected the amplitude decays exponentially for high harmonics $(n \geq 2)$. One also notes the difference between spectra taken in the near-field and the far-field regions. This difference is also expected and confirms our understanding of the formation of the cSPr in the two different zones.

To visualize the spectra observed by all the detectors, the contour plot (figure 8) has been generated. At the observation plane $(\phi=0)$, we have the following relation between the fields, 

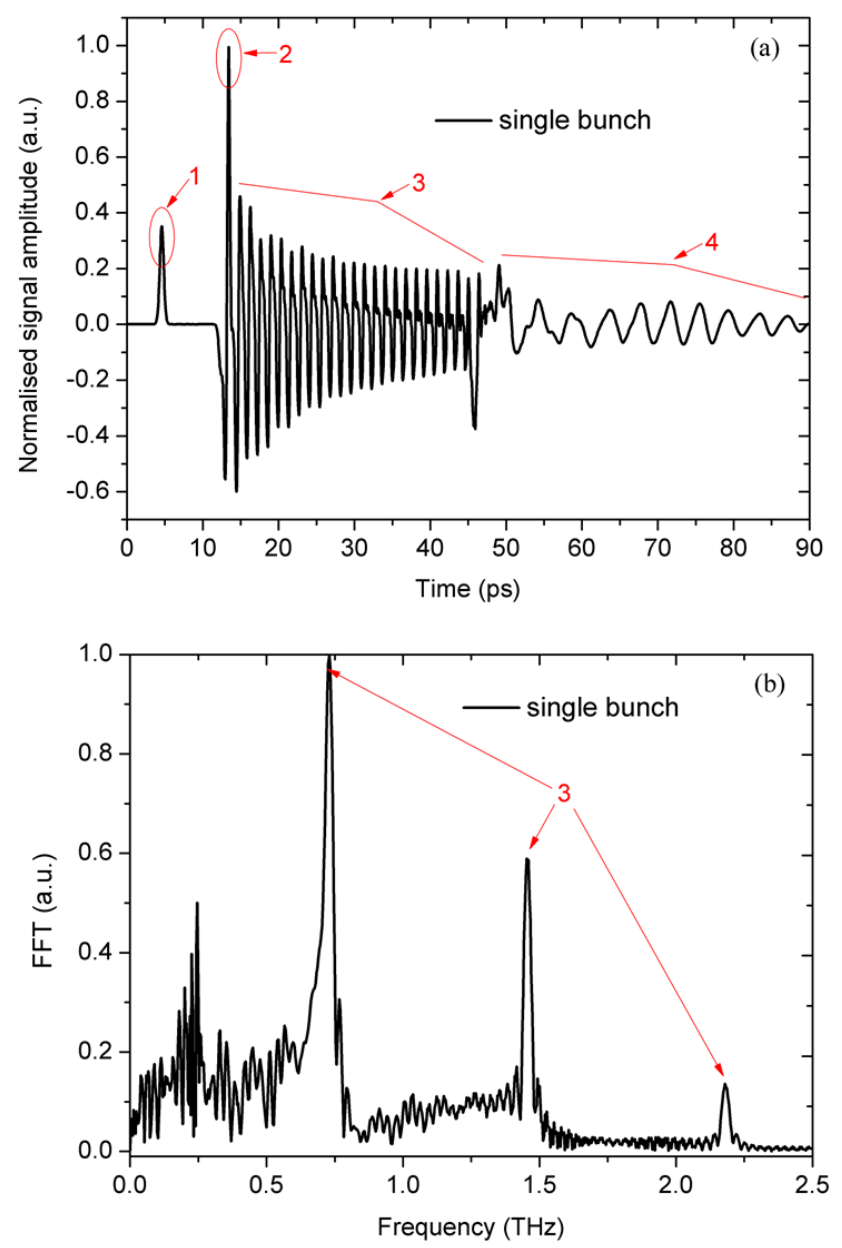

Figure 7. (a) Normalised waveform of the field $B_{\phi}(t)$ observed at $\theta=80^{\circ}$ and (b) the corresponding Fourier transform $B_{\phi}(v)$

$\boldsymbol{B}=\boldsymbol{n} \times \boldsymbol{E} / c$, where $\boldsymbol{B}=B_{\phi} \hat{\boldsymbol{e}}_{\phi}\left(B_{r}=B_{\theta}=0\right)$ and $\boldsymbol{E}=E_{\theta} \hat{\boldsymbol{e}}_{\theta}\left(E_{r}=E_{\phi}=0\right)$. The energy density spectrum at a given position in the far-field region in free space is given by [37]:

$$
\frac{d I}{d v}=\frac{2 c|\boldsymbol{B}(v)|^{2}}{\mu_{0}}
$$

Applying Fourier transforms to the cSPr signals observed at all the 91 angles and using equation 6 , the radiation spectrum in the plane $\phi=0$ was constructed in figure 8 . The dashed lines which are superimposed on the contour plot are derived from the dispersion relation (equation 1). It is clear that the Smith-Purcell radiation spectrum fits very well with the dispersion relation in the far-field region. The first harmonic $(n=1)$ of the radiation significantly decays at the frequencies around $1 \mathrm{THz}$. The radiation of the second harmonic $(n=2)$ has detectable amplitudes in the range from $0.8 \mathrm{THz}$ to $1.5 \mathrm{THz}$ while the third harmonic of the radiation is small and invisible on this plot (figure 8).

Splitting the total charge into a 4 equidistant, short microbunches and keeping other beam and grating parameters the same as before, the studies of the excitation of the cSPr 


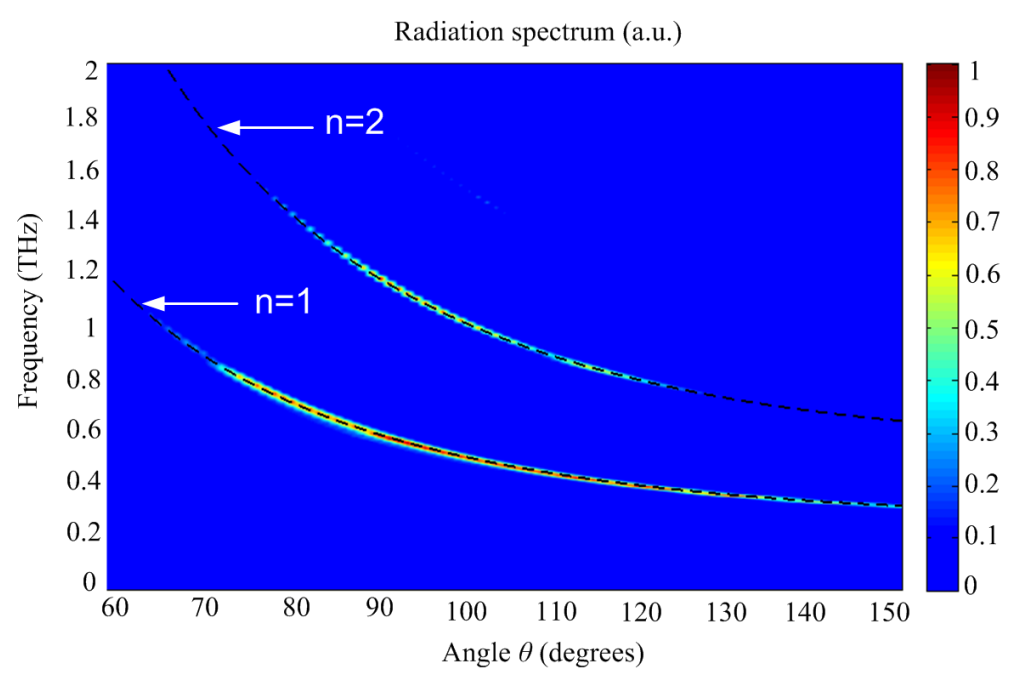

Figure 8. Normalised spectrum of the radiation generated by a single bunch as a function of angular position $\theta$ and emission frequency (observation distance $=1 \mathrm{~m}$, azimuthal angle $\phi=$ $0^{\circ}$ ). The dashed lines shows the spectra predicted by the dispersion relation in equation 1 .

by the microbunched electron beam using CST Particle Studio have been carried out. The contour plots of the spectra (similar to figure 8) of the Smith-Purcell radiation generated by the microbunch trains with the microbunch separations of $1 \mathrm{ps}$ and $1.33 \mathrm{ps}$ are shown in figures 9(a) and 9(b), respectively.

Unlike the broad Gaussian distribution generated by a single bunch (see figures 4 and 8), the radiation generated by the microbunched beams has peaks around frequencies (similar to the results observed using VSim simulations) corresponding to the spectrum picks of the microbunch train, i.e. fundamental and high harmonics of the beam current. There is a clear correlation between the microbunch periodicity and the peaks of the radiation spectra. The separations between the microbunches are $1 \mathrm{ps}$ and 1.33 ps and the spectral peaks for the fundamental harmonics are measured at $1 \mathrm{THz}$ and $0.75 \mathrm{THz}$ respectively. There is also another interesting feature shown in figure 9(a). The maximum of the radiation spectra is picked at the frequency of $1 \mathrm{THz}$, which is the second harmonic of the grating $\left(\theta \approx 102^{\circ}\right.$, $n=2$ ). This indicates that, unlike the results shown in figure 7(b) demonstrating exponential decay of the amplitudes of the harmonics with increase of their number (for a single bunch), the higher grating harmonics can be excited selectively by the train of microbunches if it is tuned accordingly. This opens the exciting opportunity to control the generation of higher harmonics of the Smith-Purcell radiation [38].

Based on the induced surface current model, a semi-analytical code named Grating of Finite Width (GFW) has been developed to describe the cSPr [35]. Considering the finite grating width of $10 \mathrm{~mm}$ and the parameters given in Table 1, the cSPr spectra have been calculated using the GFW code. The comparisons of the results from the CST simulations and calculations using the GFW code are shown in figures 9(c) and 9(d). Both the semianalytical calculations and the CST simulations predict the same position of the peaks of the radiation spectra. The agreement between the results of the numerical simulations and 

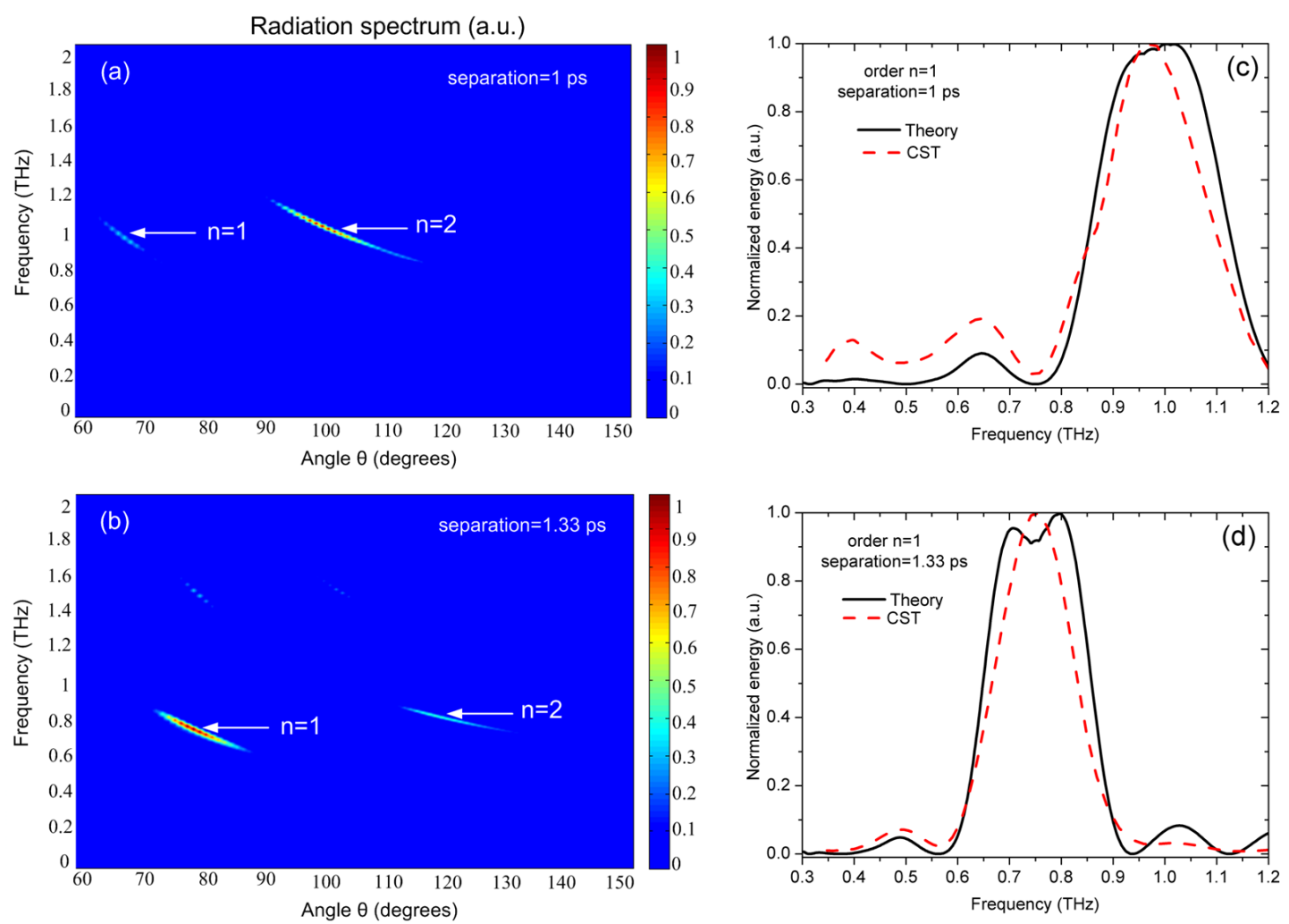

Figure 9. Radiation spectra generated by a train of a 4 identical microbunches with the separations: (a) $1 \mathrm{ps}$, (b) $1.33 \mathrm{ps}$ and obtained using the CST PS simulations. Comparison of the spectra of the cSPr observed using CST PS and GFW codes generated by the trains of 4 identical microbunches with the separations: (c) 1 ps, (d) 1.33 ps.

analytical studies is good and the differences could be attributed to the specific limitations and approximations of the methods/models used during the studies.

\section{Discussion and conclusion}

Recently some preliminary experimental work have been carried out at TTX, China, in order to study cSPr in the $\mathrm{THz}$ range. The cSPr radiation was generated from a train of the electron microbunches with the sub-ps periodicity [38]. It was shown that the maximum power of the cSPr was observed at the frequency of the microbunch spacing and the frequency selective excitation of $\mathrm{THz}$ radiation using microbunch trains was shown. A linac without energy recovery stage was used in those experiments. In order to achieve a high efficiency, the $\mathrm{THz}$ radiation source could be driven by an energy recovery linear accelerator (ERL) where the accelerated particles are recirculated back into the cavity and are hence decelerated recuperating up to $99 \%$ of the energy. The recent design of the ERL with dual axis cavity has the potential to increase the beam charge and repetition rate, making it possible to meet the demand for the energy efficient high power source of the coherent $\mathrm{THz}$ radiation. A schematic illustration of a compact THz Smith-Purcell radiation source driven by such an ERL is shown in figure 10 . 


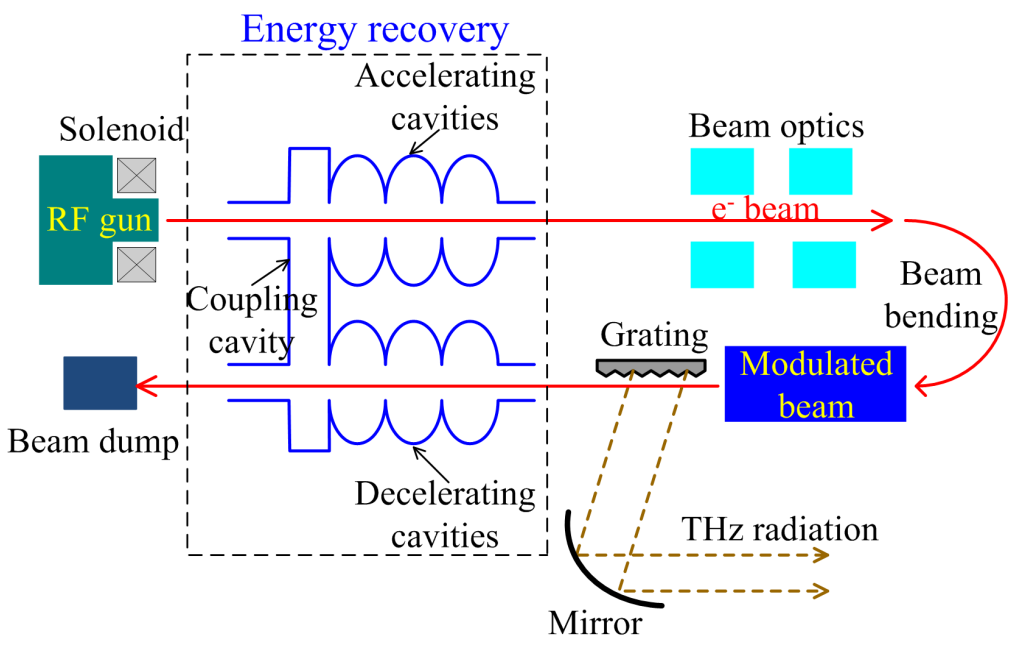

Figure 10. Schematic (not-to-scale) of the THz radiation source driven by a dual-axis ERL showing RF gun, accelerating and decelerating sections, beam optics, grating, and $\mathrm{THz}$ radiation collection/output system.

The schematics in figure 10 shows that an electron beam is emitted and accelerated from the RF gun at the first stage. The beam is driven and fully or partially modulated using one of the methods mentioned in the Introduction. The microbunched beam is used to generate coherent $\mathrm{THz}$ Smith-Purcell radiation with a mirror positioned to collect the EM radiation. Afterwards the beam goes into the decelerating section for the energy recovery $[16,17,18]$. Using available accelerator technologies such $\mathrm{THz}$ radiation source can be compact (fit in the space of $10 \mathrm{~m}^{3}$ ) and very efficient.

In conclusion, numerical studies were conducted to investigate the $\mathrm{THz}$ cSPr generated by a train of the microbunches. The studies were carried out in the near-field and the farfield regions, using the PIC software packages VSim and CST as well as semi-analytical approach. We demonstrated that if the radiation is excited by a single bunch the amplitudes of the radiation decay exponentially for high harmonics. It was shown that the microbunching of an initially 'long' (of the order of a few ps) beam results in the appearance of the peaks in the spectrum of the generated cSPr. The maximum spectral peak of the radiation coincide with the peak of the fundamental harmonic of the train of microbunches allowing a selective and efficient excitation of the high frequencies without changing the grating. This demonstrates the potential broad tunability of the radiation source by the variation of the microbunch separation and provides a method for the realization of a tunable high-power THz radiation source. Numerical results were compared with the theoretical calculations and good agreement between the results is demonstrated. The results of the studies presented and discussed will stimulate further the experimental research, developments and applications of the sources of cSPr to bridge the THz technological gap. 


\section{Acknowledgments}

The authors would like to acknowledge the partial support of the project from the STFC UK through PRD grant ST/M003590/1, the Leverhulme Trust through the International Network Grant IN-2015-012, and Royal Society - International Exchanges 2017 Cost Share Grant IEC/R3/170010.

\section{References}

[1] Dhillon S S, Vitiello M S, Linfield E H, Davies A G, Hoffmann M C, Booske J, Paoloni C, Gensch M, Weightman P, Williams G P et al. 2017 Journal of Physics D: Applied Physics 50043001

[2] Naftaly M 2015 Terahertz Metrology (Artech House)

[3] Guillet J P, Recur B, Frederique L, Bousquet B, Canioni L, Manek-Hönninger I, Desbarats P and Mounaix P 2014 Journal of Infrared, Millimeter, and Terahertz Waves 35 382-411

[4] Federici J F, Schulkin B, Huang F, Gary D, Barat R, Oliveira F and Zimdars D 2005 Semiconductor Science and Technology 20 S266

[5] Davies A G, Burnett A D, Fan W, Linfield E H and Cunningham J E 2008 Materials today 11 18-26

[6] May T, Zieger G, Anders S, Zakosarenko V, Meyer H G, Schubert M, Starkloff M, Rößler M, Thorwirth G and Krause U 2009 Proc. SPIE $730973090 \mathrm{E}$

[7] Mittleman D M, Jacobsen R H, Neelamani R, Baraniuk R G and Nuss M C 1998 Applied Physics B: Lasers and Optics 67 379-390

[8] Gerecht E, Douglass K O and Plusquellic D F 2011 Optics express 19 8973-8984

[9] Dandolo C L K and Jepsen P U 2016 Journal of Infrared, Millimeter, and Terahertz Waves 37 198-208

[10] Ishigaki K, Shiraishi M, Suzuki S, Asada M, Nishiyama N and Arai S 2012 Electronics letters 48 582-583

[11] Kawano Y 2013 Contemporary Physics 54 143-165

[12] Carr G L, Martin M C, McKinney W R, Jordan K, Neil G R and Williams G P 2002 Nature 420 153-156

[13] Bielawski S, Evain C, Hara T, Hosaka M, Katoh M, Kimura S, Mochihashi A, Shimada M, Szwaj C, Takahashi T et al. 2008 Nature Physics 4 390-393

[14] Cook A M, Tikhoplav R, Tochitsky S Y, Travish G, Williams O B and Rosenzweig J B 2009 Physical review letters 103095003

[15] Booske J H, Dobbs R J, Joye C D, Kory C L, Neil G R, Park G S, Park J and Temkin R J 2011 IEEE Transactions on Terahertz Science and Technology 1 54-75

[16] Ainsworth R, Burt G, Konoplev I and Seryi A 2016 Physical Review Accelerators and Beams 19083502

[17] Konoplev I V, Metodiev K, Lancaster A, Burt G, Ainsworth R and Seryi A 2017 Physical Review Accelerators and Beams 20103501

[18] Topp-Mugglestone M, Konoplev I V, Zhang H and Seryi A 2018 Applied Physics Letters 113243503

[19] Doucas G, Blackmore V, Ottewell B, Perry C, Huggard P G, Castro-Camus E, Johnston M B, Hughes J L, Kimmitt M F, Redlich B et al. 2006 Physical Review Special Topics-Accelerators and Beams 9092801

[20] Andrews H L, Bakkali Taheri F, Barros J, Bartolini R, Bharadwaj V, Clarke C, Delerue N, Doucas G, Fuster-Martinez N, Vieille-Grosjean M, Konoplev I V, Labat M, Le Corre S, Perry C, Reichold A and Stevenson S 2014 Physical Review Special Topics - Accelerators and Beams 17 1-13

[21] Zhang H, Konoplev I, Lancaster A, Harrison H, Doucas G, Aryshev A, Shevelev M, Terunuma N and Urakawa J 2017 Applied Physics Letters 111043505

[22] Zhang H, Konoplev I, Doucas G and Smith J 2018 Physics of Plasmas 25043111

[23] Liang Y, Du Y, Su X, Wang D, Yan L, Tian Q, Zhou Z, Wang D, Huang W, Gai W et al. 2018 Applied Physics Letters 112053501

[24] Neumann J G, Fiorito R B, OShea P G, Loos H, Sheehy B, Shen Y and Wu Z 2009 Journal of Applied Physics $\mathbf{1 0 5} 053304$

[25] Shen Y, Yang X, Carr G L, Hidaka Y, Murphy J B and Wang X 2011 Physical review letters 107204801 
[26] Zhang Z, Yan L, Du Y, Zhou Z, Su X, Zheng L, Wang D, Tian Q, Wang W, Shi J et al. 2016 Physical review letters 116184801

[27] Aryshev A, Shevelev M, Honda Y, Terunuma N and Urakawa J 2017 Applied Physics Letters 111033508

[28] Antipov S, Babzien M, Jing C, Fedurin M, Gai W, Kanareykin A, Kusche K, Yakimenko V and Zholents A 2013 Physical review letters 111134802

[29] Muggli P, Yakimenko V, Babzien M, Kallos E and Kusche K P 2008 Physical review letters 101054801

[30] Kumar N, Pukhov A and Lotov K 2010 Physical review letters 104255003

[31] Fang Y, Yakimenko V E, Babzien M, Fedurin M, Kusche K P, Malone R, Vieira J, Mori W B and Muggli P 2014 Physical review letters 112045001

[32] Smith S J and Purcell E M 1953 Physical Review 921069

[33] Brownell J H, Walsh J and Doucas G 1998 Physical Review E 571075

[34] Doucas G, Kimmitt M F, Doria A, Gallerano G P, Giovenale E, Messina G, Andrews H L and Brownell J H 2002 Physical review special topics-accelerators and beams 5072802

[35] Brownell J H and Doucas G 2005 Physical review special topics-accelerators and beams 8091301

[36] Nieter C and Cary J R 2004 Journal of Computational Physics $196448-473$

[37] Grimm O and Schmüser P 2006 10th biennial European Particle Accelerator Conference, Edinburgh 10401042

[38] Liang Y, Du Y, Wang D, Yan L, Tian Q, Chen K, Huang W, Tang C, Konoplev I, Zhang H et al. 2018 Applied Physics Letters 113171104 